\title{
Systematics of the Relationship between Vacuum Energy Calculations and Heat Kernel Coefficients
}

\author{
S. A. Fulling* \\ Department of Mathematics, \\ Texas A\&M University, \\ College Station, TX, 77843-3368 USA \\ and \\ Mathematical Sciences Research Institute, \\ Berkeley, CA, 94720-5070 USA
}

(Dated: April 3, 2003)

\begin{abstract}
Casimir energy is a nonlocal effect; its magnitude cannot be deduced from heat kernel expansions, even those including the integrated boundary terms. On the other hand, it is known that the divergent terms in the regularized (but not yet renormalized) total vacuum energy are associated with the heat kernel coefficients. Here a recent study of the relations among the eigenvalue density, the heat kernel, and the integral kernel of the operator $e^{-t \sqrt{H}}$ is exploited to characterize this association completely. Various previously isolated observations about the structure of the regularized energy emerge naturally. For over 20 years controversies have persisted stemming from the fact that certain (presumably physically meaningful) terms in the renormalized vacuum energy density in the interior of a cavity become singular at the boundary and correlate to certain divergent terms in the regularized total energy. The point of view of the present paper promises to help resolve these issues.
\end{abstract}

\footnotetext{
*Electronic address: fulling@math.tamu.edu; URL: http://www.math.tamu.edu/ fulling
} 


\section{INTRODUCTION}

The aim of this paper is to bring order and completeness into a welter of observations in the physics literature about various contributions to quantum vacuum energy (before renormalization) and their relations to the heat-kernel expansion. The primary tool is the systematic theory of Riesz means of spectral densities and their relation to the asymptotic expansions of various integral kernels associated with the central partial differential operator of the field theory (e.g., [20]); the Casimir energy, regularized by an exponential ultraviolet cutoff, is identifiable with certain terms in these expansions [18]. Both total energy and local energy density are considered; for the latter, we concentrate on the singular asymptotic behavior near a boundary, which was the subject of considerable calculational attention and physical controversy two decades ago (e.g., 12, 31]), when the Casimir effect was of great interest as a model of quantum effects in cosmology (and in the bag model of hadrons). Interest in Casimir energy has recently resurged because of greatly improved experiments [5, 33, 38, 42] and hints of technological application [9]. With it, the controversies have also reemerged (e.g., 24, 35, 36]). (More complete bibliographical citations appear later in their proper contexts.)

Section II sets up notation and summarizes the needed background information about the asymptotic expansions of integral kernels and the Riesz means of eigenvalue densities. Section III concerns the energy density of a scalar field, with emphasis on the instructive example of parallel plates with Robin boundary conditions; to avoid confusion in the quantum theory later, it is important to note that the scalar field's energy contains, already at the classical level, a term concentrated on the boundary, unless the conformal-coupling parameter is given the nonstandard value $\frac{1}{4}$. The heart of the paper is Section IV, where the structures of the asymptotic expansions of the heat kernel and the total regularized energy (as functions of an auxiliary "time") and of the renormalized energy density (as function of the distance from the boundary) are presented and then correlated in dimensions 1, 2, and 3. In Section $\nabla$ we review the controversies over infinite boundary terms in the energy and draw some conclusions on the basis of the observations in the previous section.

\section{NOTATION AND FRAMEWORK}

Let $H$ be a self-adjoint, elliptic, second-order differential operator, independent of $t$. (To avoid some extraneous complications later, let us also assume that the spectrum of $H$ is nonnegative.) Then the (hyperbolic) wave equation

$$
\frac{\partial^{2} \phi}{\partial t^{2}}=-H \phi
$$

defines a field theory with time-translation invariance. Here we consider only scalar fields, but the extension to, for instance, the electromagnetic field would be straightforward. Also, we are primarily concerned here with cavities in flat space, without potentials, so that $H$ is just the negative of the Laplacian equipped with certain boundary conditions; however, most of what follows applies with only minor complications to more general operators

$$
H=-g(x)^{\mu \nu} \nabla_{\mu}^{A} \nabla_{\nu}^{A}+V(x)
$$

with gravitational, gauge, and scalar potentials. 
For present purposes, we take the regularized vacuum energy of the quantum field to be defined as

$$
E(t) \equiv \frac{1}{2} \sum_{n} \omega_{n} e^{-\omega_{n} t},
$$

where $\omega_{n}^{2}$ are the eigenvalues of $H$, and $t$ is a parameter with dimensions of time - not the physical time in (2.1). (Of course, at this point one is assuming that $H$ has only discrete spectrum. Continuous spectrum is normally associated with an unbounded region in space, for which one would not expect a finite total energy to exist.) The physical (renormalized) energy is identified with the constant term in the asymptotic expansion of $E(t)$ as $t \rightarrow 0$, in keeping with the expectation that each mode of the field with frequency $\omega$ should contribute energy $\frac{1}{2} \omega$. Formula (2.2) is a standard, but admittedly arbitrary, and recently somewhat unfashionable, way of regularizing the energy. The relation of this and other "ultraviolet" or "cutoff" regularizations to the now more popular "analytic" methods (notably dimensional regularization and zeta functions) has been clarified by several authors 1, 11, 30, 43, 44, 45, 46].

In quantum field theory one has not only a total energy but also a local energy density, $T_{00}(x)$ (and, indeed, an energy-momentum (stress) tensor, $T_{\mu \nu}(x)$ ). The energy density remains meaningful in unbounded regions, where the total energy is not defined (and $H$ may have continuous spectrum). There is a formal expression for $T_{00}(x)$ as a sum or integral over the spectrum, the summand being built out of the eigenfunctions, or the spectral projection kernel, and their derivatives at $x$; for the details we refer to the literature (e.g., [3] ) and Sec. III From that expression a regularized quantity $T_{00}(x ; t)$ is defined by inserting an exponential cutoff in precise analogy with (2.2). In fact, it is known that this approach is not quite adequate when spatial curvature, or indeed any potential, is present, because trace anomalies arise; the conceptually closest adequate approach is then covariant point splitting (e.g., [6]). That complication is irrelevant, however, to cavities in flat space without potentials, which are our concern at present.

Let $d$ be the spatial dimension, let $M$ denote the $d$-dimensional region in question (where $H$ acts), and let $t$ be an auxiliary variable ranging from 0 to $+\infty$. The well known heat kernel, $K(t, x, y) \equiv\left\langle x\left|e^{-t H}\right| y\right\rangle$, solves the heat equation associated with $H$, in the sense that

$$
u(t, x) \equiv \int_{M} K(t, x, y) f(y) d y
$$

is the unique solution of the (parabolic) initial-value problem

$$
\frac{\partial u}{\partial t}=-H u, \quad u(0, x)=f(x) .
$$

It is well known that $K$ possesses an asymptotic expansion of the form

$$
K(t) \sim \sum_{s=0}^{\infty} b_{s} t^{-\frac{d}{2}+\frac{s}{2}} .
$$

More precisely: (a) If $x$ is a point in the interior of $M$, then the diagonal value $K(t, x, x)$ has an expansion of form (2.3), where $b_{s}$ depends on $x$. (b) When $M$ is bounded, the "trace" $\int_{M} K(t, x, x) d x$ has an expansion of form (2.3). (The second statement is not a trivial consequence of the first, because expansion (a) is nonuniform in distance from the boundary. In fact, for a flat-space cavity $b_{s}(x)$ in (a) is zero for all $s>0$, but the same 
is not true for the number $b_{s}$ in (b).) For a cavity, $b_{0}$ in (b) is proportional to the volume of $M$, and the higher coefficients are integrals over the boundary of $M$ whose details depend on the boundary conditions and on the curvature of the boundary. For a more general $H$, similar local integral formulas for $b_{s}$ in terms of the potentials, curvature, etc. inside $M$ and on its boundary are known in great detail for small values of $s$, their calculation for ever-increasing $s$ being a major mathematical industry (e.g., 32]).

The less well known cylinder kernel, $T(t, x, y) \equiv\left\langle x\left|e^{-t \sqrt{H}}\right| y\right\rangle$, can be defined similarly:

$$
u(t, x) \equiv \int_{M} T(t, x, y) f(y) d y
$$

is the unique bounded solution of the (elliptic) boundary-value problem

$$
\frac{\partial^{2} u}{\partial t^{2}}=+H u, \quad u(0, x)=f(x) .
$$

(The region in $(t, x)$-space is a semiinfinite cylinder with base $M$. .) The counterpart of (2.3) is

$$
T(t) \sim \sum_{s=0}^{\infty} e_{s} t^{-d+s}+\sum_{\substack{s=d+1 \\ s-d \text { odd }}}^{\infty} f_{s} t^{-d+s} \ln t .
$$

(Again, (2.4) has both (a)- and (b)-type interpretations.) The central fact relating these expansions (which in essence appears in [11, Sec. III]) is:

\section{Theorem.}

1. If $s-d$ is even or negative,

$$
e_{s}=\pi^{-1 / 2} 2^{d-s} \Gamma\left(\frac{d}{2}-\frac{s}{2}+\frac{1}{2}\right) b_{s}
$$

(and $\left.f_{s}=0\right)$.

2. If $s-d$ is odd and positive,

$$
f_{s}=(-1)^{(s-d+1) / 2} \pi^{-1 / 2} 2^{d-s+1} \frac{1}{\Gamma\left(\frac{s}{2}-\frac{d}{2}+\frac{1}{2}\right)} b_{s},
$$

whereas $e_{s}$ is undetermined by the heat kernel expansion.

Before explaining where this theorem comes from, we make some remarks upon it. First, in (2.5) and (2.6) the gamma functions have no zeros or poles for the relevant values of the parameters; therefore, a zero or nonzero $b_{s}$ is always associated, respectively, with a zero or nonzero value for the coefficient on the left-hand side of the equation. Second, the "new" coefficients $e_{s}(s-d$ odd and positive) are inherently global in their dependence on the geometry of $M$ : There are no local integral formulas like those for $b_{s}$ (and hence for the left-hand sides of (2.5) and (2.6) ) [22]. Third (see Sec. IV], the renormalized vacuum energy is equal to $-\frac{1}{2} e_{1+d}$ (the first of the new, nonlocal coefficients) from expansion (b), plus possible local terms. (Note that a purely local formula for vacuum energy would imply that the energy associated with parallel plates is independent of their separation, hence that there 
is no Casimir force!) Fourth, for a particular $H$ any or all of the logarithmic coefficients $f_{s}$ may happen to vanish, but the nonlocal, independent nature of the corresponding $e_{s}$ persists in such a case.

The theorem can be proved by relating the two expansions, (2.3) and (2.4), to certain "moments" (in a generalized sense of the word) characterizing the asymptotic behavior of the eigenvalue density (or of a local spectral density, the inverse Laplace transform of $K(t, x, x)$, in the "untraced" (a) situation). One suitable set of such quantities consists of residues and values, at certain points, of the zeta functions associated with the operators $H$ and $\sqrt{H}$. In that case the proof is an adaptation and generalization of the proof of Lemma 2.2 of [21]. (See also 11].) We omit the details, citing only the crucial equation

$$
\zeta_{\sqrt{H}}(2 s)=\zeta_{H}(s) .
$$

An alternative set, with more direct spectral and physical significance, consists of the Riesz means of the eigenvalue density. These are the rigorous counterpart of the formal high-frequency asymptotic expansions of the eigenvalue density whose term-by-term Laplace transforms yield the expansions (2.3) and (2.4). The importance of Riesz means in understanding (2.4), and vice versa, has been developed in [17, 19, 20]. (Papers [13, 14, 15] are related.) The present paper demonstrates the usefulness of that work in physics. We shall review it here, using the language appropriate to the "traced" (b) situation.

Riesz means [27, 28, 29] generalize the Cesàro means used in place of partial sums to accelerate the convergence of Fourier series. Let $\mu(\lambda)$ be the number of eigenvalues of $H$ less than $\lambda$ (the counting function, or "spectral staircase"). Write

$$
\lambda=\omega^{2} \quad(\omega>0) .
$$

(Thus, if $\lambda$ is an eigenvalue, $\omega$ is the frequency of the normal mode(s) of the wave equation associated with $\lambda$; also, $\omega$ is the corresponding eigenvalue of $\sqrt{H}$.) For a positive integer $\alpha$ the Riesz mean $R_{\lambda}^{\alpha} \mu(\lambda)$ is defined by averaging $\mu$ over an $\alpha$-dimensional simplex:

$$
R_{\lambda}^{\alpha} \mu(\lambda) \equiv \frac{1}{\alpha !} \lambda^{-\alpha} \int^{\lambda} \stackrel{\alpha}{\cdots} \int \mu(\tilde{\lambda}) d \tilde{\lambda},
$$

where $\alpha$ successive indefinite integrations are implied. (This operation can be reexpressed as a single integral with a nontrivial kernel function.) A different sequence of Riesz means is defined by integration over $\omega$ :

$$
R_{\omega}^{\alpha} \mu(\omega) \equiv \frac{1}{\alpha !} \omega^{-\alpha} \int^{\omega} \stackrel{\alpha}{\alpha} \int \mu\left(\tilde{\omega}^{2}\right) d \tilde{\omega},
$$

Starting from the known properties of the heat-kernel expansion (2.3), one can prove the finite asymptotic approximations

$$
\begin{gathered}
R_{\lambda}^{\alpha} \mu=\sum_{s=0}^{\alpha} a_{\alpha s} \lambda^{\frac{d-s}{2}}+O\left(\lambda^{\frac{d-\alpha-1}{2}}\right), \\
R_{\omega}^{\alpha} \mu=\sum_{s=0}^{\alpha} c_{\alpha s} \omega^{d-s}+\sum_{\substack{s=d+1 \\
s=d \text { odd }}}^{\alpha} d_{\alpha s} \omega^{d-s} \ln \omega+O\left(\omega^{d-\alpha-1} \ln \omega\right) .
\end{gathered}
$$


Here the $a$ coefficients are related quite directly to the heat-kernel coefficients:

$$
b_{s}=\frac{\Gamma\left(\frac{d+s}{2}+1\right)}{\Gamma(s+1)} a_{s s} .
$$

(The $a_{\alpha s}$ with $\alpha \neq s$ have similar formulas, which we ignore here because those coefficients contain no additional information.) Similarly, the $c$ and $d$ coefficients are related to the cylinder expansion by $\left[\psi \equiv(\ln \Gamma)^{\prime}\right]$

$$
\begin{gathered}
e_{s}=\frac{\Gamma(d+1)}{\Gamma(s+1)} c_{s s} \quad \text { if } d-s \text { is even or positive, } \\
f_{s}=-\frac{\Gamma(d+1)}{\Gamma(s+1)} d_{s s}, \quad e_{s}=\frac{\Gamma(d+1)}{\Gamma(s+1)}\left[c_{s s}+\psi(d+1) d_{s s}\right] \quad \text { if } d-s \text { is odd and negative. }
\end{gathered}
$$

The final link is the connection between $\left(c_{s s}, d_{s s}\right)$ and $a_{s s}$. In [20] the ratio of $c_{s s}$ to $a_{s s}$ was given as a complicated finite sum of gamma functions. However, hidden in the appendix of [20] (because the authors did not appreciate their significance at the time) are formulas that, after some routine manipulation, yield the relatively simple result

$$
c_{s s}=\frac{\Gamma\left(\frac{d}{2}-\frac{s}{2}+\frac{1}{2}\right) \Gamma\left(\frac{d}{2}+\frac{s}{2}+1\right)}{2^{s} \Gamma\left(\frac{d}{2}+\frac{1}{2}\right) \Gamma\left(\frac{d}{2}+1\right)} a_{s s} \text { if } d-s \text { is even or positive. }
$$

When $d-s$ is odd and negative (when the first factor in the numerator of (2.16) has a pole), 20] shows that $c_{s s}$ is undetermined by $a_{s s}$ while $d_{s s}$ comes into existence:

$$
d_{s s}=\frac{(-1)^{d+1}}{(s-d-1) ! d !} \frac{\Gamma\left(\frac{s}{2}-\frac{d}{2}\right)}{\Gamma\left(-\frac{d}{2}-\frac{s}{2}\right)} a_{s s} \quad \text { if } d-s \text { is odd and negative. }
$$

Equations (2.5) and (2.6) now follow from (2.13)-(2.17) with aid of

$$
\Gamma(z) \Gamma\left(z+\frac{1}{2}\right)=\sqrt{\pi} 2^{1-2 z} \Gamma(2 z), \quad \Gamma(-z) \Gamma(z+1)=\frac{-\pi}{\sin \pi z} .
$$

Note that the Riesz means (2.9) and (2.10) provide two families of definitions of an "averaged density of states" with certain weightings. More precisely, $R_{\lambda}^{\alpha} \mu$ or $R_{\omega}^{\alpha} \mu$ somehow describes the averaged asymptotic behavior of the eigenvalue density $\mu^{\prime}(\lambda)$ at high frequency, but it also contains constants of integration summarizing what happens to $\mu^{\prime}(\lambda)$ at low frequency. (As $\alpha$ increases, the smoothing out of the eigenvalue distribution becomes more drastic, so that the expansion (2.11) or (2.12) can be meaningfully extended to a higher order; but at the same time, the contribution from the lower frequencies becomes more dominant.) The crucial fact is that the coefficients in the omega-mean expansion (equivalently, those in the cylinder-kernel expansion) contain more information than the coefficients in the lambdamean (or the heat-kernel) expansion. Specifically, $c_{s s}$ for $s-d$ odd and positive is an independent, nonlocal spectral invariant. In particular, $s-d=1$ corresponds (modulo local terms) to the Casimir vacuum energy. 


\section{ENERGY DENSITY, ROBIN BOUNDARY CONDITIONS, AND PARALLEL PLATES}

The energy density of a scalar field in flat space-time is (e.g., [3] )

$$
T_{00}=\frac{1}{2}\left[\left(\frac{\partial \phi}{\partial t}\right)^{2}+(1-4 \xi)(\nabla \phi)^{2}-4 \xi \phi \nabla^{2} \phi\right],
$$

where $\xi$ is an arbitrary number called the conformal coupling constant. In curved spacetime $\xi$ derives from a term (in the Lagrangian or the equation of motion) coupling $\phi$ to the curvature; in a gravitational context, therefore, different values of $\xi$ correspond to different physical theories. In flat space, however, $\xi$ does not appear in the equation of motion, nor in the spectral decomposition of $H$, and the terms proportional to $\xi$ in (3.1) form a total divergence, $-2 \xi \nabla \cdot(\phi \nabla \phi)$. The facile conclusion is that all values of $\xi$ yield the same total energy and hence are physically equivalent. We shall see, however, that subtleties arise at both classical and quantum levels.

Because it leads to nontrivial phenomena even in one dimension, it is instructive to study the Robin boundary condition,

$$
\frac{\partial \phi}{\partial n}=\gamma \phi
$$

Here $\gamma(x)$ is, in general, a function defined on the boundary of the region $M$, and $\frac{\partial}{\partial n}$ is the outward normal derivative. Dimensionally, $\gamma$ is an inverse length. Romeo and Saharian [41] have recently made an exhaustive study of the Casimir-like situation of two parallel plates with a constant value of $\gamma$ on each plate. (A continuing series of papers by Saharian and coworkers address similar issues in more complicated geometries). Of course, the special cases of the Neumann $(\gamma=0)$ and Dirichlet (formally, $\gamma=\infty$ ) boundary conditions have been understood for a long time. With the sign convention in (3.2), our condition of nonnegative spectrum for $H$ is guaranteed by taking $\gamma$ negative; Romeo and Saharian consider both signs of $\gamma$ (which they call $\beta^{-1}$ ), ignoring the contributions to the energy from modes with negative eigenvalues, which do not affect the renormalization theory.

If $M$ is bounded, one should be able to integrate $T_{00}$ over $M$ to obtain a conserved energy. (For parallel plates, integration in the perpendicular dimension yields an energy per unit plate area.) In fact, in the Robin theory it is necessary to include in the energy a surface term concentrated on the boundary:

$$
E \equiv \int_{M} T_{00}(x) d^{d} x+\frac{1}{2}(4 \xi-1) \int_{\partial M} \gamma \phi^{2} d S
$$

(where $d S$ is the element of $(d-1)$-dimensional surface area on the boundary, $\partial M)$. A calculation, using the equation of motion (2.1) for $\phi$ and an integration by parts, shows that $\frac{\partial E}{\partial t}=0$, the boundary term being essential for this result if $\gamma \neq 0$ and $\xi \neq \frac{1}{4}$. Such a term was apparently introduced into quantum field theory - through a different rationale - in 31]. Classically, the term is presumably related to the fact that a one-dimensional Robin problem models a vibrating string attached to a point mass, which can exchange energy with the string (e.g., [10, p. 15]).

Three things should be noted about the boundary term in (3.3). First, it is a completely classical matter; in particular, it is not a renormalization counterterm (at least, not yet). Second, it arises only in the full Robin theory: it vanishes in the pure Neumann case $(\gamma=0)$ 
and the Dirichlet case $(\phi=0$, where the conservation of $E$ without that term can be easily verified). Third, even in the Robin case it vanishes if $\xi=\frac{1}{4}$. Changing $\xi$ (in flat space) amounts to redefining some of the boundary energy as spread throughout the interior of $M$ by integration by parts, and $\frac{1}{4}$ is the choice that moves the energy entirely into the interior. The striking thing about this value of $\xi$ is that it is neither the "minimal" choice $(\xi=0)$ nor the choice that makes the full theory, including gravitation, conformally invariant. The "conformal" value is well known to be

$$
\xi_{\mathrm{c}} \equiv \frac{d-1}{4 d}
$$

which equals 0 when $d=1$, equals $\frac{1}{6}$ when $d=3$, and approaches $\frac{1}{4}$ as $d \rightarrow \infty$.

In the quantum field theory one evaluates the expectation values of observables such as $T_{00}(x)$ and $E$ in the vacuum, or ground state, of the field. The general rule of thumb [3] for doing so, when the observable is classically defined by a quadratic form $T(\phi, \phi)$, is to evaluate the associated bilinear form on the normal modes of the field and sum:

$$
\langle 0|T| 0\rangle=\sum_{n} T\left(\tilde{\phi}_{n}, \tilde{\phi}_{n}^{*}\right) .
$$

Here $\tilde{\phi}(t, x)=\left(2 \omega_{n}\right)^{-1 / 2} \phi_{n}(x) e^{-i \omega_{n} t}$, where $\phi_{n}$ is an eigenvector of $H$ with eigenvalue $\omega_{n}^{2}$; generalization to continuous spectrum is possible. (Henceforth the vacuum bra-ket symbols will be omitted when there is no danger of confusion.) The result of this formal calculation is usually a divergent sum or integral, which must be regularized - perhaps by inserting an exponential ultraviolet cutoff, exactly as in (2.2) - and ultimately renormalized. (However, the calculations of energy density in [41] do not require an explicit regularization step; instead, the renormalization is accomplished by isolating and subtracting at the integrand stage the terms corresponding to the vacuum state in infinite flat space.) When such calculations were done some 20-30 years ago for the Dirichlet and Neumann problems with parallel plates, it was found that when $\xi=\xi_{\mathrm{c}}$ the (renormalized) energy density $T_{00}(x)$ is constant (and nonzero) everywhere between the plates, thus yielding a finite Casimir energy (per unit area) that must be attributed to the global configuration of the plates, not to an interaction between the field and the boundary per se. (In the Neumann case the contribution of the mode with $\omega=0$ is being ignored here. When a zero-frequency mode exists, there is no true vacuum state, but vacuum conditions can be approached arbitrarily closely.) On the other hand, for $\xi \neq \xi_{\mathrm{c}}$ the renormalized energy density includes a contribution strongly concentrated near the plates themselves; that part is, in fact, nonintegrable, hence at least superficially inconsistent with a finite value for the renormalized total energy. When the boundary is curved, there are such near-boundary terms even when the coupling constant takes the conformal value, although the singularity at the boundary is weaker in that case. The recent Robin results [1] are a consistent extension of that picture:

- For a single (flat) plate, the renormalized $T_{00}$ vanishes if $\xi=\xi_{\mathrm{c}}$, regardless of $\gamma$.

- In general, the energy density around a single plate is proportional to $\xi-\xi_{\mathrm{c}}$. If $x$ is distance from the plate, $T_{00}(x)$ is proportional to $x^{-(d+1)}$ in the Dirichlet and Neumann cases, and in the general Robin case it has additional terms proportional to $\gamma^{k} x^{k-(d+1)}$ $(k=1, \ldots, d)$ and $\ln |\gamma x|$. (Romeo and Saharian actually find an exact expression for $T_{00}$, of which these terms are merely the divergent part of the asymptotic expansion for small $x$.) 
- For two parallel plates, $T_{00}$ is constant in the space between the plates if $\xi=\xi_{\mathrm{c}}$, with a rather complicated dependence on the parameters $a \gamma_{1}$ and $a \gamma_{2}$ ( $a$ being the plate separation and $\gamma_{i}$ the Robin coefficients on the two plates), which reduces for the pure Dirichlet or Neumann problem to the known $a^{-(d+1)}$ dependence.

- For parallel plates with $\xi \neq \xi_{\mathrm{c}}$, the result is even more complicated, but it can be written as the sum of the effects of the individual plates (described above) plus an interaction term that is everywhere finite.

- Romeo and Saharian perform an independent calculation of the renormalized total energy by the zeta-function method. This calculation is independent of $\xi$, as the eigenvalues do not depend on $\xi$. The result includes a term that depends logarithmically on an arbitrary scale parameter — indicating that a logarithmic divergence would appear in some other regularization methods. This term is independent of $a$, hence presumably unobservable.

- The authors demonstrate a certain formal consistency between their energy-density and total-energy calculations. In particular, for a single plate they find

$$
E_{\mathrm{vol}} \equiv \int_{0}^{\infty} T_{00}(x) d x=4 d\left(\xi-\xi_{\mathrm{c}}\right) E, \quad E_{\mathrm{surf}} \equiv \frac{1}{2}(4 \xi-1) \gamma\left\langle 0\left|\phi(0)^{2}\right| 0\right\rangle=d(1-4 \xi) E,
$$

where $E=E_{\mathrm{vol}}+E_{\text {surf }}$ is the putative total energy per unit area (on one side) of the plate. However, they point out that the integrals defining the three quantities in (3.6) are actually divergent. One can eliminate the volume term by taking $\xi=\xi_{\text {c }}$, or one can eliminate the surface term by taking $\xi=\frac{1}{4}$, but not both at once.

In view of the last point, a strong case can be made that the most convenient choice of $\xi$ is $\frac{1}{4}$, rather than the traditional values, 0 or $\xi_{\mathrm{c}}$. One can then avoid accounting for and renormalizing the surface term, and one can be confident that any volume contribution must be taken seriously, not dismissed as an artifact.

We have summarized the paper [41] at some length because the parallel-plate Robin system is such an excellent, explicitly solvable model of the phenomena and issues that arise in more general systems, notably cavities with curved boundaries. The principal extrinsic curvatures of a boundary surface have the dimension of inverse length and enter calculations or expansions of energy density and total energy in very much the same way as does the Robin coefficient $\gamma$. The leading divergence in $T_{00}$ as the boundary is approached is ubiquitous when $\xi$ does not have its conformal value; unlike the Dirichlet case, the classical surface term in the total energy of the Robin system interferes with the facile dismissal of this problem as some kind of artifact of a poor definition of energy density (whereas the nonexistence of the surface term in the Dirichlet case shows that the problem can't be resolved by a simple cancellation of volume and surface contributions, either). The higher-order terms in the expansion of $T_{00}$ near the boundary, which appear for any $\xi$ when $\gamma \neq 0$, are closely analogous to terms that appear near curved boundaries for the electromagnetic field, as well as the conformally coupled scalar one.

\section{SCHEMATICS OF THE EXPANSIONS}

Consider now a general cavity whose boundary is characterized by an extrinsic curvature tensor, $\kappa(x)$, and also equipped with a Robin function, $\gamma(x)$. The structure of the heat 
expansion (2.3), for the trace, is

$$
K(t) \sim t^{-d / 2}\left[V+S t^{1 / 2}+(\kappa+\gamma) t+\left(\kappa^{2}+\gamma^{2}+\kappa \gamma\right) t^{3 / 2}+\left(\kappa^{3}+\cdots\right) t^{2}+\cdots\right] .
$$

Here, and in all similar equations later, all the terms are indicated very schematically: all numerical coefficients, the integrations over $\partial M$, and the tensorial nature of $\kappa$ when $d \geq 3$ are suppressed, as are terms involving derivatives of $\gamma$ or $\kappa$ (which are not important in low orders and dimensions). $V$ is the volume of $M$, and $S$ is the surface area of $M$ (more precisely, the $(d-1)$-dimensional volume of $\partial M)$.

The schematic form of the (traced) cylinder expansion (2.4) now follows by the theorem in Sec. II] The significance of the structure depends on the dimension. In the following formulas, the coefficients in boldface are the new, nonlocal terms, $e_{s}$, and those in square brackets are constant terms, which do not contribute to the regularized energy,

$$
E(t)=-\frac{1}{2} \frac{d T}{d t}
$$

(The trace of the operator $e^{-t \sqrt{H}}$ is $\sum_{n} e^{-\omega_{n} t}$, so (4.2) is equivalent to (2.2).)

$$
d=1: \quad T(t) \sim V t^{-1}+[S]+\gamma t \ln t+\mathbf{E} t+\gamma^{2} t^{2}+\gamma^{3} t^{3} \ln t+\mathbf{F} t^{3}+\cdots .
$$

(In dimension 1 there is no curvature, and the "surface area" $S$ degenerates to the number of endpoints of the region - usually 2. The $\gamma$ terms are also sums over the endpoints.)

$$
\begin{array}{rlrl}
d=2: & T(t) & \sim V t^{-2}+S t^{-1}+[\kappa+\gamma]+\left(\kappa^{2}+\gamma^{2}+\kappa \gamma\right) t \ln t+\mathbf{E} t+\left(\kappa^{3}+\cdots\right) t^{2}+\cdots \\
d=3: & T(t) \sim V t^{-3}+S t^{-2}+(\kappa+\gamma) t^{-1}+\left[\kappa^{2}+\gamma^{2}+\kappa \gamma\right]+\left(\kappa^{3}+\cdots\right) t \ln t+\mathbf{E} t+\cdots
\end{array}
$$

The untraced kernel expansions can be discussed similarly, but there is less to say. In the cavity setting, where there are no potentials or spatial curvature, the local heat expansion is trivial:

$$
K(t, x, x) \sim t^{-d / 2}+O\left(t^{\infty}\right) .
$$

The local cylinder expansion, therefore, has the structure

$$
T(t, x, x) \sim t^{-d}+\mathbf{E}(x) t+\cdots .
$$

Let $T_{00}(t, x)$ be the energy density, regularized by an exponential cutoff as discussed below (3.5). The relation between $T_{00}(t, x)$ and $T(t, x, x)$ is less straightforward than that between their global counterparts, $E(t)$ and $T(t)$. The analogue of (4.2) yields only the terms in (3.1) involving time derivatives. The other terms can be obtained [18] by applying appropriate spatial partial derivatives to the integral kernel of the operator

$$
\frac{1}{\sqrt{H}} e^{-t \sqrt{H}}
$$

which has not been as extensively studied as the cylinder operator but has a very similar nature. (Note that the original cylinder kernel is the time derivative of this new one.) For the qualitative considerations of the present paper it is not necessary to delve further into the details; it is not misleading to think of $T_{00}(t, x)$ as "essentially" the $t$-derivative of $T(t, x, x)$.

This is as far as the heat kernel can take us. To find $\mathbf{E}(x)$ one must do independent calculations. But the literature, notably [12, 31, 41], provides considerable information 
about the behavior of the renormalized $T_{00}(x)$ as $x$ approaches a boundary. Our task now is to compare these expansions in distance from the boundary (which we once again denote by $x$ ) to the expansions (in $t$ ) of the regularized total energy, the derivatives of (4.3) - (4.5). The point is that the finite, renormalized energy density generally is not integrable up to the boundary, and if such divergent terms are taken seriously (and not compensated by postulated terms concentrated exactly on the boundary [31], whose discussion we postpone to Sec. $(\nabla)$, then for consistency they must somehow be reflected in terms of $E(t)$ that become infinite in the limit $t \rightarrow 0$ and hence would be removed in a conventional renormalization of the energy.

\section{A. Dimension 1}

The energy density found by Romeo and Saharian [41] near a Robin endpoint (with $\left.\xi=\frac{1}{4}\right)$ can be correlated with the derivative of (4.3) as follows:

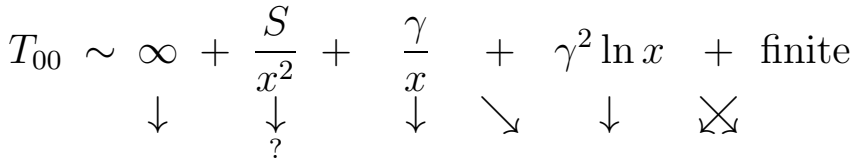

$$
\begin{aligned}
& E \sim \frac{V}{t^{2}} \quad+\gamma \ln t+(\mathbf{E}+\gamma)+\gamma^{2} t+\cdots
\end{aligned}
$$

The leading $\infty$ in $T_{00}$ is the universal, $x$-independent formal vacuum energy of infinite empty flat space, which everyone agrees should be discarded in renormalization. It is included here because it clearly corresponds directly to the volume divergence in $E$. The arrows indicate how other terms in the two series might be related, on the basis of their dependence on $\gamma$. (Whether all these arrows represent genuinely existing relationships remains to be investigated by a thorough and rigorous calculation of $T_{00}(t, x, x)$, followed by an integration over $x$ with $t$ finite but approaching 0 . That project is beyond our present scope.) The most striking observation is that the "surface" term in the density has completely disappeared from the total energy. (Recall that the corresponding term in (4.3) was destroyed by the differentiation.) The $1 / x$ term in the density might be held responsible for the $\ln t$ term in the energy, as well as part of the finite term. The $\ln x$ term is integrable, and its integral is presumably included in E. Finally, we note that all the explicitly $\gamma$-dependent terms in $E$ will be independent of the length of the cavity, so they will not contribute to the Casimir force between the endpoints (usually considered the only quantity of experimental relevance). The force must come entirely from $\mathbf{E}$.

\section{B. Dimension 2}

From now on, for brevity, the Robin function, $\gamma$, will not be explicitly indicated in the displays (4.10) and (4.11), because it enters in the same way as the curvature, $\kappa$. I do not know a reference for the dependence of $T_{00}(x)$ upon $\kappa$ in dimension 2 , but it appears to be dictated by dimensional analysis and analogies with the Robin formula [4] and the 
three-dimensional results [12].

$$
\begin{aligned}
& \begin{array}{ccccccc}
T_{00} \sim & \infty \\
\downarrow & +\frac{S}{x^{3}}+\frac{\kappa}{x^{2}}+ & \frac{\kappa^{2}}{x} & & & \kappa^{3} \ln x & + \text { finite } \\
\downarrow & \downarrow & \downarrow & \searrow & \downarrow & \searrow
\end{array} \\
& E \sim \frac{V}{t^{3}}+\frac{S}{t^{2}} \quad+\kappa^{2} \ln t+\left(\mathbf{E}+\kappa^{2}\right)+\kappa^{3} t+\cdots
\end{aligned}
$$

Most noteworthy here are the term $\kappa^{2} / x$ in the density and the corresponding term $\kappa^{2} \ln t$ in the total energy. The analogous terms in dimension 3 are proportional to $\kappa^{3}$. When total energy calculations are done for both the inside and the outside of a boundary surface (a "shell" modeling a thin electrical conductor), it has often been noted that divergent contributions proportional to odd powers of $\kappa$ will cancel, because $\kappa$ has opposite signs on the two sides. This fact gives rise to an important difference between even and odd dimensions. In even dimensions, the $\ln t$ term in $E$ is proportional to an even power of $\kappa$, so it survives even for thin shells. Logarithmic terms in $E(t)$ regularized by ultraviolet cutoff are precisely the divergences that persist in analytic regularization schemes, giving rise, under even the most liberal interpretation [4], at least to ambiguous finite terms in the renormalized energy that depend logarithmically on an arbitrary mass scale. In dimensional regularization, the $\kappa^{d}$ divergence shows up as a pole at each even value of $d$ in the total Casimir energy associated with a spherical shell [35]. Here we have seen in a more direct, elementary way that such a term is dictated by the known form of the heat kernel.

\section{Dimension 3}

We are now in the domain of the classic results of Deutsch and Candelas [12] and Kennedy, Critchley, and Dowker [31], which also apply to the electromagnetic field.

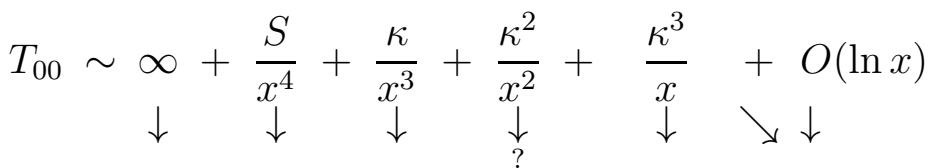

$$
\begin{aligned}
& E \sim \frac{V}{t^{4}}+\frac{S}{t^{3}}+\frac{\kappa}{t^{2}} \quad+\kappa^{3} \ln t+\left(\mathbf{E}+\kappa^{3}\right)+\cdots
\end{aligned}
$$

Let us review the litany of special features [7, 12] that make the Casimir force on a spherical conducting shell finite, even when calculated by integrating the energy density right up to the boundary: The volume term is removed by the local renormalization. The surface term vanishes for electromagnetism because of a cancellation between electric and magnetic contributions. The $\kappa$ and $\kappa^{3}$ terms cancel (between inside and outside) for any thin shell. That leaves $\kappa^{2}$. In dimension $2, \kappa$ is a symmetric matrix with two eigenvalues, $\kappa_{1}$ and $\kappa_{2}$ (the principal curvatures). There are thus two independent quadratic invariants to be integrated over $\partial M$, which may be chosen as

$$
D \equiv \kappa_{1} \kappa_{2}, \quad \Delta \equiv\left(\kappa_{1}-\kappa_{2}\right)^{2} .
$$

For a sphere, $\Delta$ is identically zero. For any closed surface, $\int_{\partial M} D(x) d S$ is a topological invariant by the Gauss-Bonnet theorem, so its derivative with respect to the sphere's radius vanishes. (More generally, for any bounded region $M$, any quadratic integral of the type 
$\int_{\partial M} \kappa^{2} d S$ is constant under a shape-preserving magnification of $M$, even if $\Delta$ is involved. Note that this observation does not settle how the energy per unit length of an infinitely long cylinder depends on the radius, since the scaling of the area is different in that case.) As emphasized by Candelas [7, 8, 12], one would nevertheless expect infinite energy differences to result from shape-changing deformations of a closed surface, because of the $\Delta$ term. It is therefore significant that no term of the type $\kappa^{2} / t$ can appear in the regularized total energy, $E(t)$, because of the structure of the traced cylinder expansion, (2.4) or (4.5). We return to this issue in the next section.

\section{IMPLICATIONS}

The theory of vacuum energy in electromagnetism has increasing experimental verification and many indirect implications for observable phenomena. Nevertheless, the simplest and crispest theoretical models tend to be idealizations that are not directly testable. Perhaps for this reason, controversies persist about renormalization procedures and the physical significance of various divergences. These disputes fall into two main categories.

First, there is the broad question of whether "logarithmic" divergences are a sign of a bad model, or just an inherent renormalization ambiguity. Among the divergences that arise in energies regularized by point-splitting or ultraviolet cutoffs, such as the second line of (4.11), only those proportional to $\ln t$ survive [4, 11, 30] in analytic regularization schemes, where they appear as poles at the physical values of the regularization parameters. The analytic methods therefore give finite energies automatically in many problems, without a need for a renormalization subtraction, but in some other problems are embarrassed by a surviving divergence whose removal introduces an unavoidable ambiguity parametrized by an arbitrary length or mass scale. Some authors [4, 31] regard this as not at all a problem, while others regard the poles as "truly disturbing" [35, p. 199] or at least puzzling.

Second, and specific to Casimir calculations, is the question of whether the nonintegrable divergences in the renormalized energy density near boundaries must be taken seriously, even in cases when the renormalized total energy (or the observable force) is finite. In fact, how can such a divergence even be regarded as logically consistent with a finite total energy? Both Kennedy et al. 31] and Milton and coworkers [34, 35, 37] trust the calculations of finite total energies and forces more than the calculations of local energy densities. Kennedy et al. argued that the infinities in $T_{00}(x)$ must be compensated by delta-function terms concentrated exactly on the boundary itself, so that the finite total energy predicted by zetafunction calculations will result. (The boundary term in (3.3) originated in this discussion in [31], without a clear explanation of how it could be relevant when $\xi=\frac{1}{4}$ or $\gamma=0$ or $\infty$.) Milton writes [35, p. 237], “... [T] he local vacuum-fluctuation energy density is to a large extent meaningless... . [D]ivergences in the energy which go like a power of the cutoff are probably unobservable, being subsumed in the properties of matter."

On the other side, Candelas and Deutsch [7, 8, 12] argued that the boundary infinities in the energy density are real (within the theory) and must be regarded as a defect of an overly idealized model. This position has recently been reexpressed by Graham, Jaffe, and coworkers [23, 24, 25, 26, 39]. The argument, stated in electromagnetic terms, is that no real material is perfectly conducting at arbitrarily high frequencies. When an idealized boundary is replaced by a more realistic model of the interaction of the quantized field with other matter, the infinite pileup of energy near the boundary will become finite, but possibly very large and definitely physically real. A strong argument for this position is that 
the energy-momentum tensor acts as the source of the gravitational field, so a nonintegrable singularity in it is physically unacceptable, even if the total energy comes out finite by virtue of a cancellation (as happens in some scenarios involving thin shells).

The present paper does not resolve all these issues, but it casts some light on them and suggests some directions for future research that will clarify them further, as indicated in the following remarks.

\section{A. Thin shells; Robin divergences}

Much has been made of the disappearance of some divergences by cancellation in the case of thin shell boundaries. The dimensional dependence (even vs. odd) of this effect is quite clear from (4.9)-(4.11) and their antecedents. Those formulas also indicate analogous divergences specific to boundaries with nontrivial Robin coefficients, which were discovered in [41] but could have been anticipated from an understanding of the relation between vacuum energy and the heat kernel. Note that there is no reason to expect any cancellation of exterior and interior Robin effects for a thin boundary.

\section{B. Disappearing $x^{-2}$ terms; total derivatives; conductors with anisotropic curva- ture}

A pervasive feature of (4.9)-(4.11) is that terms in $T_{00}$ proportional to $x^{-2}$ ( $x$ being distance to the boundary) never have counterparts in the regularized total energy. On grounds of dimensional analysis such terms would be expected to appear in $E(t)$ and be proportional to $t^{-1}$, but the derivation of $E(t)$ from the heat and cylinder kernels shows that they are not there! (The crucial fact is that terms proportional to $t^{n} \ln t$ occur in the cylinder kernel only if $n$ is odd and positive - in particular, not for $n=0$.) This absence of

$t^{-1}$ terms was noted by Cognola et al. [11], and very recently by Bernasconi et al. 2], who found that it persists when the exponential cutoff is replaced by a Gaussian one.

In dimension 1 the $x^{-2}$ term is precisely the one associated with nonconformal coupling - that is, the leading boundary divergence, which (in any dimension, but for a scalar field) does not occur for $\xi=\xi_{\mathrm{c}}$ and arises for other values of $\xi$ from a term proportional to $\left(\xi-\xi_{\mathrm{c}}\right) \nabla \cdot(\phi \nabla \phi)$ in the formal energy density (cf. (3.1) and ensuing discussion). Since this term is a total derivative, it ought to give 0 when integrated. Effectively this seems to be true, since the renormalized total energy is finite and independent of $\xi$. As has been recognized (if not "understood") since [12] and [31], integration over $x$ somehow does not commute with renormalization. There is a simpler and completely understood analogous situation with the heat kernel: The local heat expansion (4.6) is nonuniform in $x$, and hence integrating it over $M$ does not yield the correct trace expansion, (4.11). The interesting terms in the latter come from a part of $K(t, x, x)$ that concentrates entirely on the boundary in the limit $t \rightarrow 0$. It is tempting to think of the boundary terms in $T_{00}$ (see (4.9)-(4.11)) as the cylinderkernel analogues of these boundary terms in $K$, but they are not: they are nonvanishing even when $t=0$. More likely, there is some other part of $T(t, x, x)$ that concentrates right on the boundary in the limit and partly or wholly compensates the integrals of the energy divergences near the boundary. This would corroborate, within the framework of ultraviolet regularization, the proposal of Kennedy et al. [31], made in the framework of zeta-function regularization. (It would not, however, solve the problem of a singular Einstein equation.) 
We propose, therefore, a program to calculate $T(t, x, x)$ in various models for finite $t$ and to integrate it over $x$, and then to examine the limit $t \rightarrow 0$. The expectation is that the results (including contributions from the kernel of (4.8) if necessary) will be fully consistent with the direct regularization and renormalization of the total energy, and that the details of the process will demonstrate precisely how the terms in the expansion of the renormalized $T_{00}(x)$ near the boundary are related, consistently, with the divergent terms in $E(t)$. Some steps in this program can already be extracted from the literature: Parentani [40] has made a similar observation in the context of a uniformly accelerating boundary. Ford and Svaiter [16], also with an eye to resolving the paradox of the total energy of the nonconformally coupled scalar field, studied a statistically fluctuating boundary. Their formulas seem mathematically identical to the results of ultraviolet regularization with an arbitrary (not necessarily exponential) cutoff function, with a fluctuation parameter in the place of $t$. They find (in dimension 3 ) that the nonconformal term of $T_{00}$ oscillates from negative to positive and back to negative, in such a way its integral is indeed zero. Finally, Graham et al. 23, 24, 26, 39] replace the ideal boundary with an extended object modeled by a second quantized field. Their results are very similar to those of [16] in dimension 3 and have one fewer oscillation in dimension 2. (Olum and Graham [39] also point out that the boundary itself, as a physical object, has an energy density of its own that can be expected to swamp the energy induced in the scalar field; nevertheless, they conclude that the total energy density remains negative in a small region displaced slightly from the boundary.) All the available results suggest that the cancellation is insensitive to the functional form of the ultraviolet cutoff, and also to its physical interpretation (fluctuating boundary, soft boundary, or mere mathematical device).

In dimensions greater than 1 the leading boundary divergence associated with a nonconformal stress tensor does not go as $x^{-2}$, so the additional boundary terms that render the regularized energy $\xi$-independent are not related (at least, not directly) to the $x^{-2}$-versus- $t^{-1}$ phenomenon. Instead, that phenomenon would appear to have some other implications. In particular, in dimension 3 it indicates that $E$ contains no term proportional to $\Delta=\left(\kappa_{1}-\kappa_{2}\right)^{2}$. This conclusion seems to be robust against changes in the form of the ultraviolet cutoff [2]. But local density calculations indicated to Candelas and Deutsch [7, 8, 12] that that term would be not only large but negative (persisting into the renormalized energy for a physical conductor), thereby rendering a conducting foil unstable against wrinkling. This may well be true for a physical, imperfect conductor. However, in the framework of formal ultraviolet regularization of the idealized theory, our conjecture is that there is a compensating positive term that concentrates right on the boundary as the regularization parameter is taken to 0 , thereby removing the apparent conflict between local and global calculations. Clearly, detailed calculations are needed to verify this conjecture and to establish its relevance to realistic conductors. (Incidentally, the sharp-eyed modern reader may notice that the original argument concerning the $\Delta$ term in Appendix A of [12] incorrectly assumed that it is correlated with a term of order $\omega^{-1}$ in a formal expansion of the counting function $\mu$. Indeed, Candelas pointed out in a later paper [7, Sec. 7.3] that no such term exists in $\mu$, but that its alleged effects can still arise in the integrated contribution of the oscillatory part of the eigenvalue distribution. In the terminology of our Sec. II the relevant terms in the cylinder kernel and in $T_{00}$ are associated with constants of integration in Riesz means rather than with the local behavior of $\mu^{\prime}(\lambda)$, or even $\mu(\lambda)$, at large $\lambda$. Therefore, this tangential technical issue in no way invalidates the physical conclusions of [12].) 


\section{Genuine surface effects}

On the other hand, almost certainly there are some real near-boundary energies. In (4.9)(4.11) one observes terms of order $x^{-1}$ in the renormalized energy density, which appear to be related to the divergent terms of order $\ln t$ in the regularized total energy (although explicit calculations, of the sort described above, should be performed to confirm this). As previously mentioned, terms of the latter type are agreed to yield terms with inherently ambiguous coefficients in the renormalized energy. Also, terms of order $\ln x$ in $T_{00}$ are integrable and presumably contribute part of the finite term in $E$.

\section{Nonrelativistic Casimir energy?}

On p. 30 of [35], Milton raises the question of why, mathematically, there is no Casimir energy in nonrelativistic quantum theory. (Physically, such energy can't exist because the relativistic effect vanishes exponentially with large field mass.) He mimics the relativistic zeta-function calculation — for parallel plates with Dirichlet conditions — and finds an expression containing a gamma function in the denominator, whose pole forces the quantity to vanish in the relevant limit. The same conclusion can be reached more transparently from our point of view. The formal energy is

$$
E=\frac{1}{2} \sum_{n} \frac{\omega_{n}^{2}}{2 m}
$$

which can be regularized in analogy with (4.2) as

$$
\frac{1}{4 m} \frac{d^{2}}{d t^{2}} \sum_{n} e^{-t \omega_{n}} .
$$

But the sum here is simply $T(t)$, which has, as in (4.9)-(4.11), the schematic form

$$
T(t) \sim \text { divergent terms }+\mathbf{E} t+\left(\kappa^{d+1}+\cdots+\gamma^{d+1}\right) t^{2}+\mathbf{F} t^{3}+\cdots,
$$

the coefficient of the $t^{2}$ term being dictated by the heat-kernel expansion. Therefore, in the renormalized energy one gets

$$
\left.\frac{d^{2} T}{d t^{2}}\right|_{t=0}=0
$$

because $\kappa$ and $\gamma$ are zero in the system studied.

In summary, what we have shown is that various results for specific models that previously emerged from forests of gamma and Riemann zeta functions now are easy consequences of the known general forms of the heat and cylinder expansions. There are, to be sure, enough gamma functions in [20] and Sec. II] to satisfy anyone, but they are handled once and for all in the general theory, rather than arising in each concrete calculation out of some physically opaque analytic continuation. With respect to the controversies over global surface-energy renormalization versus local energy-density calculations, we have not taken a position squarely on either side. Rather, we have presented evidence that each side is correct in certain respects. In particular, a mechanism of partial surface cancellation has been pointed out that shows promise of reconciling the two points of view. For the leading 
boundary divergence of the scalar field, this mechanism has been fairly well established by other researchers [16, 26, 40]; for the $x^{-2}$ divergence, it is conjectured by the present author as the only visible way of restoring consistency between the mathematical structures of the local and global asymptotic expansions.

\section{Acknowledgments}

This material was first presented at the Workshop on Casimir Forces at the HarvardSmithsonian Center for Astrophysics; I am grateful to the organizers for the invitation and to many of the participants, especially Larry Ford, Nami Svaiter, and Kim Milton, for comments and bibliographical information. The manuscript was written during a semester at MSRI, and I acknowledge the financial generosity of both that Institute and my home department that made that stay possible. Joel Bondurant aided in the study of the energy density and its boundary term in their dependence on $\xi$ and $\gamma$.

[1] C. G. Beneventano and E. M. Santangelo, Connection between $\zeta$ and cutoff regularizations of Casimir energies, Internat. J. Mod. Phys. A 11, 2871-2886 (1996).

[2] F. Bernasconi, G. M. Graf, and D. Hasler, The heat kernel expansion for the electromagnetic field in a cavity, math-ph/0302035

[3] N. D. Birrell and P. C. W. Davies, Quantum Fields in Curved Space, Cambridge U. Press, Cambridge, 1982.

[4] S. K. Blau, M. Visser, and A. Wipf, Zeta functions and the Casimir energy, Nucl. Phys. B 310, 163-180 (1988).

[5] G. Bressi, G. Carugno, R. Onofrio, and G. Ruoso, Measurement of the Casimir force between parallel metallic surfaces, Phys. Rev. Lett. 88, 041804 (2002).

[6] T. S. Bunch, S. M. Christensen, and S. A. Fulling, Massive quantum field theory in twodimensional Robertson-Walker space-time, Phys. Rev. D 18, 4435-4459 (1978).

[7] P. Candelas, Vacuum polarization in the presence of dielectric and conducting surfaces, Ann. Phys. (NY) 143, 241-295 (1982).

[8] P. Candelas, Vacuum energy in the bag model, Ann. Phys. (NY) 167, 257-284 (1986).

[9] H. B. Chan, V. A. Aksyuk, R. N. Kleiman, D. J. Bishop, and F. Capasso, Quantum mechanical actuation of microelectromechanical systems by the Casimir force, Science 291, 1941-1944 (2001).

[10] G. Chen and J. Zhou, Boundary Element Methods, Academic Press, London, 1992.

[11] G. Cognola, L. Vanzo, and S. Zerbini, Regularization dependence of vacuum energy in arbitrarily shaped cavities, J. Math. Phys. 33, 222-228 (1992).

[12] D. Deutsch and P. Candelas, Boundary effects in quantum field theory, Phys. Rev. D 20, 3063-3080 (1979).

[13] R. Estrada, The Cesàro behaviour of distributions, Proc. Roy. Soc. Lond. A 454, 2425-2443 (1998).

[14] R. Estrada and S. A. Fulling, Distributional asymptotic expansions of spectral functions and of the associated Green kernels, Electronic J. Diff. Eqs. 1999, No. 7, 1-37 (1999). 
[15] R. Estrada, J. M. Gracia-Bondía, and J. C. Várilly, On summability of distributions and spectral geometry, Commun. Math. Phys. 191, 219-248 (1998).

[16] L. H. Ford and N. F. Svaiter, Vacuum energy density near fluctuating boundaries, Phys. Rev. D 58, 065007 (1998).

[17] S. A. Fulling, What we should have learned from G. H. Hardy about quantum field theory under external conditions, in The Casimir Effect Fifty Years Later, ed. by M. Bordag, World Scientific, Singapore, 1999, pp. 145-154.

[18] S. A. Fulling, Periodic orbits, spectral oscillations, scaling, and vacuum energy: Beyond HaMiDeW. Nucl. Phys. B (Proc. Suppl.) 104, 161-164 (2002).

[19] S. A. Fulling, E. V. Gorbar, and C. T. Romero, Spectral Riesz-Cesaro means: How the square root function helps us to see around the world, Electron. J. Diff. Eqs., Conf. 04, 87-101 (2000).

[20] S. A. Fulling and R. A. Gustafson, Some properties of Riesz means and spectral expansions, Electronic J. Diff. Eqs. 1999, No. 6, 1-39 (1999).

[21] P. B. Gilkey, The spectral geometry of the higher order Laplacian, Duke Math. J. 47, 511-528 (1980).

[22] P. B. Gilkey and G. Grubb, Logarithmic terms in asymptotic expansions of heat operator traces, Commun. Partial Diff. Eqs. 23, 777-792 (1998).

[23] N. Graham, R. L. Jaffe, V. Khemani, M. Quandt, M. Scandurra, and H. Weigel, Calculating vacuum energies in renormalizable quantum field theories: A new approach to the Casimir problem, Nucl. Phys. B 645, 49-84 (2002).

[24] N. Graham, R. L. Jaffe, V. Khemani, M. Quandt, M. Scandurra, and H. Weigel, Casimir energies in light of quantum field theory, hep-th/0207205

[25] N. Graham, R. L. Jaffe, and H. Weigel, Casimir effects in renormalizable quantum field theories, Internat. J. Mod. Phys. A 17, 846-869 (2002).

[26] N. Graham and K. D. Olum, Negative energy densities in quantum field theory with a background potential, hep-th/0211244

[27] G. H. Hardy, The second theorem of consistency for summable series, Proc. London Math. Soc. (2) 15, 72-88 (1916).

[28] L. Hörmander, On the Riesz means of spectral functions and eigenfunction expansions for elliptic differential operators, in Belfer Graduate School of Science Annual Science Conference Proceedings: Some Recent Advances in the Basic Sciences, Vol. 2 (1965-66), ed. by A. Gelbart, Yeshiva University, New York, 1969, pp. 155-202.

[29] L. Hörmander, The spectral function of an elliptic operator, Acta Math. 121, 193-218 (1968).

[30] B. S. Kay, Casimir effect in quantum field theory, Phys. Rev. D 20, 3052-3062 (1979).

[31] G. Kennedy, R. Critchley, and J. S. Dowker, Finite temperature field theory with boundaries: Stress tensor and surface action renormalization, Ann. Phys. (NY) 125, 346-400 (1980).

[32] K. Kirsten, Spectral Functions in Mathematics and Physics, Chapman \& Hall/CRC, Boca Raton, 2002.

[33] S. K. Lamoreaux, Demonstration of the Casimir force in the 0.6 to $6 \mu \mathrm{m}$ range, Phys. Rev. Lett. 78, 5-8 (1997).

[34] K. A. Milton, Semiclassical electron models: Casimir self-stress in dielectric and conducting balls, Ann. Phys. (NY) 127, 49-61 (1980).

[35] K. A. Milton, The Casimir Effect: Physical Manifestations of Zero-Point Energy, World Scientific, Singapore, 2001.

[36] K. A. Milton, Calculating Casimir energies in renormalizable quantum field theory, 
hep-th/0210081.

[37] K. A. Milton, L. L. DeRaad, and J. Schwinger, Casimir self-stress on a perfectly conducting spherical shell, Ann. Phys. (NY) 115, 388-403 (1978).

[38] U. Mohideen and A. Roy, Precision measurement of the Casimir force from 0.1 to $0.9 \mu \mathrm{m}$, Phys. Rev. Lett. 81, 4549-4552 (1998).

[39] K. D. Olum and N. Graham, Static negative energies near a domain wall, gr-qc/0205134.

[40] R. Parentani, The energy-momentum tensor in Fulling-Rindler vacuum, Class. Quantum Grav. 10, 1409-1415 (1993).

[41] A. Romeo and A. A. Saharian, Casimir effect for scalar fields under Robin boundary conditions on plates, J. Phys. A 35, 1297-1320 (2002).

[42] A. Roy and U. Mohideen, Demonstration of the nontrivial boundary dependence of the Casimir force, Phys. Rev. Lett. 82, 4380-4383 (1999).

[43] E. M. Santangelo, Evaluation of Casimir energies through spectral functions, Theor. Math. Phys. 131, 527-542 (2002) [Teor. Mat. Fiz. 131, 98-117 (2002)]; the preprint version, hep-th/0104025, is recommended, as the published English version has suffered from reverse translation.

[44] B. F. Svaiter and N. F. Svaiter, Zero point energy and analytic regularizations, Phys. Rev. D 47, 4581-4585 (1993).

[45] N. F. Svaiter and B. F. Svaiter, Casimir effect in a $D$-dimensional flat space-time and the cut-off method, J. Math. Phys. 32, 175-180 (1991).

[46] N. F. Svaiter and B. F. Svaiter, The analytic regularization zeta function method and the cut-off method in the Casimir effect, J. Phys. A 25, 979-989 (1992). 